\title{
Reject analysis in digital radiography: A local study on radiographers and students' attitude in Iran
}

\author{
Sajjad Rastegar ${ }^{1,2}$, Jalal Beigi ${ }^{1,2}$, Ehsan Saeidi ${ }^{1,2}$, Ali Dezhkam ${ }^{1,2}$, Tofigh Mobaderi ${ }^{3}$ \\ Hamed Ghaffari*4,5, Ali Mehdipour ${ }^{2}$, Hamid Abdollahi*6 \\ Received: 11 Dec 2018 \\ Published: 29 May 2019
}

\begin{abstract}
Reject analysis is as a quality indicator and critical tool for dose and image quality optimization in radiology departments. By reducing image rejection rate (RR), radiation dose to patients can be reduced effectively, yielding increased total cost-effectiveness. The aims of this study were to assess the rate of image rejection at 2 direct digital radiography (DR) departments to find the sources of rejection and to observe how radiology students and radiographers deal with image rejection.

Two radiology departments were surveyed during a 3-month period for all imaging procedures. Type of examination, numbers, and reasons for digital image rejection were obtained by systems and questionnaire. A predefined questionnaire, including 13 causes for rejection, was filled by radiographers and students.

Out of the 14022 acquired images, 1116 were rejected, yielding an overall RR of 8\%. Highest RRs were found for examination of cervical spine and lumbosacral. Positioning errors and improper patient preparation were the main reasons for digital image rejection. The image RR was small, but there is a need for optimizing radiographic practice, and enhancing radiographer's knowledge may enhance the performance.
\end{abstract}

Keywords: Digital radiography, Image rejection, Quality assurance, Radiation safety

Conflicts of Interest: None declared

Funding: None

\section{*This work has been published under CC BY-NC-SA 1.0 license. \\ Copyright $\odot$ Iran University of Medical Sciences}

Cite this article as: Rastegar S, Beigi J, Saeidi E, Dezhkam A, Mobaderi T, Ghaffari H, Mehdipour A, Abdollahi H. Reject analysis in digital radiography: A local study on radiographers and students' attitude in Iran. Med J Islam Repub Iran. 2019 (29 May);33:49. https://doi.org/10.47176/mjiri.33.49

\section{Introduction}

Film reject analysis (RA) has been considered as an important component of quality assurance (QA) program in radiology departments for many years (1). Also, film RA is used to determine the extent to which as low as reasonably achievable (ALARA) principle is adhered in radiology units (2). Reject/retake of diagnostic x-ray images impose several important consequences within radiological imaging, including an unnecessary radiation exposure to

Corresponding author: Dr Hamid Abdollahi, hamid_rbp@yahoo.com Hamed Ghaffari, hamedghaffari@yahoo.com

1. Student Research Committee, Paramedical Faculty, Rafsanjan University of Medical Sciences, Rafsanjan, Iran

2. Department of Radiology Technology, Paramedical Faculty, Rafsanjan University of Medical Sciences, Rafsanjan, Iran

3. Department of Biostatistics, School of Health, Iran University of Medical Sciences, Tehran, Iran

4. Department of Medical Physics, School of Medicine, Iran University of Medical Sciences, Tehran, Iran

5. Student Research Committee, School of Medicine, Iran University of Medical Sciences, Tehran, Iran

6. Department of Radiologic Sciences and Medical Physics, Faculty of Allied Medicine, Kerman University of Medical Sciences, Kerman, Iran patient, occupational costs such as radiology staff time, the cost of purchasing and maintaining processing chemicals, and the cost of purchasing x-ray films (3-6). In filmbased radiology departments, reject/retake rates range between $10 \%$ and $15 \%(5)$, and the most important reason can be attributed to incorrect exposure conditions owing to limited dynamic range of screen/film systems. However, the advent of new radiological technologies, such as

\section{$\uparrow$ What is "already known" in this topic:}

Film reject analysis is a main component of quality assurance program at a film-based radiology department. The number of rejected films can be an indicator of image quality, and it also shows that as low as reasonably achievable (ALARA) principle is adhered in radiology units. The rejection rate of images can reduce with digitalization of medical images.

\section{$\rightarrow$ What this article adds:}

With the advent of new radiology technologies, such as direct digital radiography, the rejection rate of images did not fall to zero. Positioning errors and improper patient preparation were main reasons for rejection. 
computed radiography (CR), direct digital radiography (DR), and the digitalization of medical imaging, can reduce the reject/retake rates. Studies have shown that even with digitalization of medical imaging, the rejection rate did not fall to zero due to mispositioning, improper collimation, examining a wrong patient, wrong exposures, etc. (7). In recent years, many studies have been published on RA in digital radiology for QA and on reducing unnecessary radiation exposure, processing, and occupational costs. Studies have shown that rate of image rejection has been decreased by digital systems and many of source of errors have been eliminated $(5,8)$.

A study by Khafaji et al obtained a RR of $15 \%$ in DR departments (3). Foos et al reported a RR of $4.4 \%$ in CR departments that seem to be an optimized value (9). In another study, Andersen et al evaluated radiographer reasons for image rejection and found that positioning errors has the highest reason for image rejection in DR departments and reported a RR of $12 \%(6)$.

In Iran, in addition to radiographers who work alone or as a team, radiology technology students are trained in academic hospitals under the supervision of expert and experienced trainers. They perform imaging procedures in their last 2 years of education course. Students have a scientific relationship with radiographers and learn from them. Although student's imaging is supervised by their trainers, they may reject images alone. However, radiographers can independently make a decision to reject images.

In the present study, it was aimed to assess the rate of image rejection at 2 direct DR departments, to find the sources of rejection, and to observe how radiology students and personnel deal with image rejection. This study was conducted in 2 academic general hospitals in Rafsanjan, a city in southeast of Iran. To the best of our knowledge, no similar study has been done in Iran.

This prospective study was approved by the ethics committee of Rafsanjan University of Medical Sciences. Also, permission was obtained from the Head of Radiology Departments. No repeats were taken for the purpose of the present study. Also, patients' information was not recorded.

Data for reject analysis were collected during a 3-month period (July-September 2017) from 2 direct DR departments at 2 local hospitals in Rafsanjan, Iran. Both hospitals were general and perform all radiology examinations for outpatients and hospitalized patients. These hospitals are operative $24 / 7$. The x-ray laboratories that were included in the present study had similar equipment (installed 2007, X-ray tube by Sedecal, model Millennium Plus, with detector model DRX-CS wireless $(43 \times 43$ $\mathrm{cm})$ ).

In these departments, radiology personnel work as team or alone; and students are trained under the supervision of 3 expert trainers. The staffs decide to keep or reject images immediately after exposure alone or consult with one another. Image rejections for students are based on trainers' decision directly or consulting with experienced personnel. All rejected and accepted images were sent to Picture Archiving and Communication System (PACS) and were recorded along with the name of the relevant radiographer.

To analyze image RR and main its causes, all rejected images were counted and the relevant radiographer was questioned. To simplify RR analysis, 13 predefined causes, including artifacts caused by jewelry, wrong use of the equipment, artifacts caused by equipment errors, poor inspiration, incorrect information in the referral, low dose, patient motion, positioning errors, additional picture, and improper patient preparation were provided. To analyze radiographers and students' attitudes against image rejection, a questionnaire was distributed to them (Table 1). Also, the items listed in the rejection causes and questionnaire (Table 1) were developed based on existing literature $(3,4,6,8)$. Radiographers and students participated in the present study voluntarily.

A total of 14022 digital images were taken during a 3month period. Of them, 1116 were rejected during this period (Table 2). The number of acquired images, rejected images, and the RRs for the different imaging examinations are presented in Table 2. The results showed an overall RR of $8 \%$. The chest, knee, ankle, pelvic, foot and wrist, as most frequently occurring examinations, had reject rates of $5 \%, 4 \%, 0 \%, 17 \%, 3 \%$ and $3 \%$, respectively.

A total of 18 personnel and 27 students completed the questionnaire on rejection attitude. The answers to the questions are summarized in Table 1 . About $6 \%$ of the personnel had less than 1 year and $17 \%$ had more than 10 years of radiography experience. About $66 \%$ of radiographers had a range between 1 and 5 years of radiography experiences.

Moreover, $78 \%$ of personnel reported that they have enough experience to reject the images and $22 \%$ believed that it is always easy to select a reason for rejection (Table 1). However, $72 \%$ of students reported that they have enough experience to reject the images and $18 \%$ believed that it is always easy to select a reason for rejection. Nevertheless, only $15 \%$ of students rejected images without their trainers' decision or personnel support.

The distribution of identified reasons for image rejection is outlined in Table 1 . In response to scenarios of suboptimal image quality, the selection of reasons by both radiographers and students had a large variety, and positioning errors and improper patient preparation were the main reasons for rejection. As displayed in Table 1, 67\% and $70 \%$ of the personnel and students expressed that they have not worked with automatic exposure control (AEC) an $\mathrm{x}$-ray exposure termination device, respectively.

\section{A brief discussion on results and comparison with} similar studies in the world

To the best of our knowledge, this was the first study on digital image rejection analysis in Iran. The total number of rejected images during the study period was 1116 and the overall RR was $8 \%$, which can be considered as an acceptable RR. The reject rate obtained from this study is in line with reject range obtained from similar studies in digital departments. With a CR system, a RR of $6.6 \%$ and $7.3 \%$ were reported $(4,10)$. In good agreement with both 
Table 1. Question and scenarios given to radiographers to assess variation in the selection of the reasons for rejection

\begin{tabular}{|c|c|c|c|c|c|}
\hline \multirow[t]{2}{*}{ Questions } & \multirow[t]{2}{*}{ Alternatives } & \multicolumn{2}{|c|}{ Radiographer } & \multicolumn{2}{|c|}{ Student } \\
\hline & & $\begin{array}{l}\text { Answers } \\
\text { frequency }\end{array}$ & $\begin{array}{l}\text { Answer } \\
\text { percent }\end{array}$ & $\begin{array}{l}\text { Answers } \\
\text { frequency }\end{array}$ & $\begin{array}{l}\text { Answer } \\
\text { percent }\end{array}$ \\
\hline & $<1$ & 1 & 6 & - & - \\
\hline \multirow{2}{*}{$\begin{array}{l}\text { rapher? } \\
\text { rapers }\end{array}$} & $5-10$ & 2 & 11 & & \\
\hline & $>10$ & 3 & 17 & & \\
\hline \multirow[t]{4}{*}{ What is your opinion of reject analysis? } & Good & 1 & 6 & 2 & 7 \\
\hline & Not so good & 13 & 72 & 18 & 67 \\
\hline & Do not know & 0 & 0 & 0 & 0 \\
\hline & Yes, it is always easy & 4 & 22 & 5 & 18 \\
\hline \multirow[t]{3}{*}{ Is it easy to select a reason for rejection? } & No, sometimes it is difficult & 13 & 72 & 16 & 60 \\
\hline & No, it is always difficult & 1 & 6 & 5 & 18 \\
\hline & $\begin{array}{l}\text { Have never selected a reason for } \\
\text { rejection }\end{array}$ & 0 & 0 & 1 & 4 \\
\hline Do you feel that you have received adequate training & Yes & 14 & 78 & 20 & 74 \\
\hline for performing rejection analysis? & No & 4 & 22 & 7 & 26 \\
\hline or with the help of their trainers or personnel? & With trainer/personnel & - & & 23 & 85 \\
\hline
\end{tabular}

What reason would you choose for rejecting the images in the following scenarios?

You forgot the required angularity of the tube for a specific procedure.

You have taken a chest x-ray of an elderly audioimpaired man. The patient was unsteady during the examination and the resulting image showed poor inspiration and the left sinus was not projected. An image of a young child being held by its parent is acquired. In the obtained image the parents' fingers are projected onto the image.

As part of the follow-up of a patient with a shoulder fracture an x-ray image is acquired. Prior to the examination the previous images are not reviewed. The image reveals a more distal fracture and a new image using a humorous procedure is required.

A lateral chest $\mathrm{x}$-ray is acquired using automatic exposure control (AEC). Due to the presence of soft tissue within the field of view the image is underexposed.

Positioning error
Image artefacts
Improper patient preparation
Additional image
Positioning error
Poor inspiration
Patient movement
Positioning error
Image artefact
Patient movement
Improper patient preparation
Additional picture
Positioning error
Improper patient preparation
Incorrection information in the
referral

$\begin{array}{cc}11 & 61 \\ 3 & 17 \\ 3 & 17 \\ 1 & \\ 4 & 22 \\ 13 & 72 \\ 1 & 6 \\ 0 & \\ 0 & \\ 4 & 0 \\ 7 & 22 \\ 7 & 39 \\ 1 & 39 \\ 4 & 22 \\ 13 & 72\end{array}$

\begin{tabular}{ccc}
61 & 16 & 59 \\
17 & 4 & 15 \\
17 & 5 & 18 \\
5 & 2 & 8 \\
22 & 6 & 22 \\
72 & 18 & 67 \\
6 & 3 & 11 \\
& & \\
0 & 0 & 0 \\
0 & 2 & 8 \\
22 & 8 & 30 \\
39 & 9 & 31 \\
39 & 9 & 31 \\
6 & 2 & 7 \\
22 & 5 & 18 \\
72 & 20 & 75 \\
& & \\
0 & & 0 \\
6 & 0 & 0 \\
27 & 0 & 70 \\
67 & 8 & \\
\hline
\end{tabular}

Honea and Andersen's findings, the present work revealed that the main cause for rejection was mispositioning $(6,7)$.

In this study, it was found that digital image rejection is a challenging issue and it is not easy to find a feasible reason to reject images. Although the radiographers responded to all answers, there was a lack of knowledge among them to find a reasonable cause for image rejection. On the other hand, the student's response to image rejection analysis was strongly dependent on their trainers, and only a few students could reject the images alone.

There are several reasons for image rejection, including positioning errors, image artefacts, improper patient preparation, and additional images. In addition, wrong exposure is considered as a reason for image rejection which alters imaging features as biomarkers for various clinical applications (11). In this study, the results revealed that the amount of image rejection may change based on the work experience of radiographers and patients' characteristics. Previous studies have also indicated that RRs change by patient population, type of examination performed, the equipment used, how the rejections are registered, and the skills of the radiographers $(7,12-14)$.
The results showed a low RR in many of examinations which indicates that there is no urgent need for optimizing radiographic practice. As shown in Table 2, the most rejected examinations were cervical spine and lumbosacral. Based on our experience, inappropriate positioning is the most cause of rejection in both cases with direct DR system. Therefore, radiographers at 2 centers may need more training in both examinations (cervical spine and lumbosacral). In the present study, more than $94 \%$ of radiographers had more than 1 year of work experience, which could be the reason for low RR. Our data showed that RR was less than previous studies in film-based departments, and the current study confirms that image digitalization can reduce RR (7).

In this study, radiology students' attitude for image rejection was in line with that of the personnel. Although they were under training and not allowed to make independent decisions, it seemed that they had enough and good knowledge to reject the images.

Authors have considered several limitations for this study. First, there was no audit for image analysis and radiographers evaluated the image and the reason for re- 
Table 2. Rejects during a 3-month period in radiology centers

\begin{tabular}{lccc}
\hline Examination & Images $(\mathrm{n})$ & Rejected images (n) & Rejection rate* $(\%)$ \\
\hline Abdomen & 432 & 90 & 21 \\
Elbow & 774 & 27 & 3 \\
Ankle & 1656 & 0 & 0 \\
Pelvic & 1611 & 270 & 17 \\
Cervical spine & 351 & 162 & 46 \\
Foot & 1602 & 45 & 3 \\
Hip & 198 & 9 & 5 \\
Hand & 783 & 18 & 2 \\
Wrist & 1350 & 45 & 3 \\
Knee & 2007 & 90 & 4 \\
Lumbosacral & 387 & 171 & 44 \\
Shoulder & 585 & 0 & 0 \\
Chest & 3456 & 189 & 5 \\
\hline Rejection rate (RR) for individual organ examinations was calculated by dividing the number of rejected images by the total number of acquired images
\end{tabular}

jection, which might have affected the results. Second, the number of radiographers and departments evaluated were small. Thus, further study with large data may result in better performance and RR analysis. Third, the radiographers could remove images directly and not send them to PACS. In this situation, a specific software may improve the results.

In conclusion, a RR of $8 \%$ was found for digital radiography in this study. Also, the results showed that RR is comparable to that of previous studies; however, the cause of rejection may be different. Positioning errors and improper patient preparation were the main reasons for rejection. Highest RRs were found for cervical spine and lumbosacral examinations, and thus further optimization may be required. Moreover, it was found that radiographers varied in image rejection decision making, and this study suggests a regular reject analysis as a part of QA program at radiology departments.

\section{Acknowledgements}

The authors sincerely thank Mr. Mustafa Pakdaman and Radiology Personnel at Ali-Ibn-Abitaleb hospital for their kind and helpful collaboration in this study.

\section{Conflict of Interests}

The authors declare that they have no competing interests.
9. Foos DH, Sehnert WJ, Reiner B, Siegel EL, Segal A, Waldman DL. Digital radiography reject analysis: data collection methodology, results, and recommendations from an in-depth investigation at two hospitals. J Digit Imaging. 2009; 22(1):89-98.

10. Prieto C, Vano E, Ten JI, Fernandez JM, Iñiguez AI, Arevalo N, et al. Image retake analysis in digital radiography using DICOM header information. J Digit Imaging. 2009; 22(4): 393-399.

11. Saeedi E, Dezhkam A, Beigi J, Rastegar S, Yousefi Z, Mehdipour LA, et al. Radiomic Feature Robustness and Reproducibility in Quantitative Bone Radiography: A Study on Radiologic Parameter Changes. J Clin Densitom. 2018. doi: 10.1016/j.jocd.2018.06.004.

12. Nol J, Isouard G, and Mirecki J. Digital repeat analysis; setup and operation. J Digit Imaging. 2006;19(2):159-166.

13. Nol J, Isouard G, and Mirecki J. Uncovering the causes of unnecessary repeated medical imaging examinations, or part of, in two hospital departments. Radiographer. 2005;52(3):26-31.

14. Jones AK, Heintz P, Geiser W, Goldman L, Jerjian K, Martin M, et al. Ongoing quality control in digital radiography: Report of AAPM Imaging Physics Committee Task Group 151. Med Phys. 2015;42:6658-70

\section{References}

1. Jabbari N, Zeinali A, Rahmatnezhad L. Patient dose from radiographic rejects/repeats in radiology centers of Urmia University of Medical Sciences, Iran. Health. 2012;4(2):94-100.

2. Abdollahi H, Shiri I, Salimi Y, Sarebani M, Mehdinia R, Deevband $\mathrm{MR}$, et al. Radiation dose in cardiac SPECT/CT: An estimation of SSDE and effective dose. Eur J Radiol. 2016;85(12):2257-2261.

3. Khafaji MA, Hagi SK. Direct digital radiograph. Technicians role in obtaining good images. Saudi Med J. 2014;35(8):879-881.

4. Waaler D, Hofmann B. Image rejects/retakes--radiographic challenges. Radiat Prot Dosimetry. 2010;139(1-3):375-9.

5. Peer S, Peer R, Giacomuzzi SM, Jaschke W. Comparative reject analysis in conventional film-screen and digital storage phosphor radiography. Radiat Prot Dosimetry. 2001;94(1-2):69-71.

6. Andersen ER, Jorde J, Taoussi N, Yaqoob SH, Konst B, Seierstad T. Reject analysis in direct digital radiography. Acta Radiologica. 2012;53(2):174-178.

7. Honea R, Elissa Blado M, and Ma Y. Is reject analysis necessary after converting to computed radiography? J Digit Imaging. 2002;15:41-52.

8. Hofmann B, Rosanowsky TB, Jensen C, Wah KH. Image rejects in general direct digital radiography. Acta Radiol Open. 2015;4(10):1-6. 